\title{
LOCALIZATION IN A PRINCIPAL RIGHT IDEAL DOMAIN
}

\author{
RAYMOND A. BEAUREGARD
}

\begin{abstract}
Let $R$ be a principal right ideal domain with right $D$-chain $\left\{R^{(\alpha)} \mid 0 \leqq \alpha \leqq \delta\right\}$, and let $K_{\alpha}=R\left(R^{(\alpha)}\right)^{-1}$ be the associated chain of quotient rings of $R$. The local skew degree of $R$ is defined to be the least ordinal $\lambda$ such that $K_{\lambda}$ is a local ring. The main result states that for each $\alpha \geqq \lambda, K_{\alpha}$ is a local ring; equivalently, $R$ has a unique $(\alpha+1)$-prime for $\delta>\alpha \geqq \lambda$.
\end{abstract}

In this note we consider conditions under which certain right quotient rings of a principal right ideal domain (PRI domain) are local rings. A PRI domain is a (not necessarily commutative) integral domain with unity in which each right ideal is a principal right ideal. By a local ring we understand a ring with unity in which the set of nonunits is closed under addition. Thus a local ring may be characterized as a ring with unity containing a unique maximal (right or left) ideal, namely the set of nonunits of the ring. A local PRI domain is then a PRI domain that has a unique prime (a nonzero nonunit that has no proper factorizations).

Before stating our main result we review some of the preliminaries that may be found in [1] and the references given there. A subset $S \neq \varnothing$ of nonzero elements of an integral domain $R$ is a right quotient monoid in $R$ if

(1) $a b \in S$ iff $a, b \in S(a, b \in R)$,

(2) $a \in S, b \in R$ implies $a R \cap b S \neq \varnothing$.

Then the set $K=R S^{-1}=\left\{r \mathrm{~s}^{-1} \mid r \in R, s \in S\right\}$ can be made into a ring in the usual way. Addition and multiplication in $K$ are carried out by using the fact that $a^{-1} b=b_{1} a_{1}^{-1}$ for each $a \in S, b \in R$ by (2). The ring $K$ is the right quotient ring of $R$ with respect to $S ; \mathbf{1}$ it is an integral domain with unity with the property that the units of $K$ that belong to $R$ are precisely the members of $S$. It is easily established that $K$ is a local ring iff $R \backslash S$ is an ideal of $R$. For each right ideal $A$ of $R, A S^{-1}$ is a right ideal of $K$; on the other hand, if $B$ is a right ideal of $K$ then $B=(B \cap R) S^{-1}$

Received by the editors January 4, 1971.

AMS 1969 subject classifications. Primary 1615.

Key words and phrases. PRI domain, $D$-chain, primes, infinite primes, localization.

${ }^{1}$ In general, the right quotient ring of $R$ with respect to $S$ is defined with (1) replaced by the weaker condition that $S$ be multiplicatively closed.

(C)American Mathematical Society 1972 
and $B \cap R$ is a right ideal of $R$. From this it follows that if $R$ is a PRI domain then so is $K$.

For the remainder of this paper $R$ is a PRI domain. Examples of right quotient monoids in $R$ include the group $U_{R}$ of units of $R$, and the monoid $R^{*}$ of nonzero elements of $R$. Since prime factorizations in $R$ are unique (up to order of factors and similarity) the dimension $\operatorname{dim}(a)$ of $a \in R^{*}$ can be defined to be $n \geqq 0$ if $a$ is the product of $n$ primes, and $\infty$ otherwise. It is shown in [1] that $R^{\prime}=\left\{a \in R^{*} \mid \operatorname{dim}(a)<\infty\right\}$ is a right quotient monoid in $R$.

Any PRI domain $R$ has a right $D$-chain. This means that there exists an ordinal $\delta$ and a chain $\left\{R^{(\alpha)} \mid 0 \leqq \alpha \leqq \delta\right\}$ of right quotient monoids in $R$ satisfying the following conditions in which $K_{\alpha}$ denotes the right quotient ring $R\left(R^{(\alpha)}\right)^{-1}$ :

(1) $R^{(0)}=U_{R}, R^{(\delta)}=R^{*}$,

(2) $R^{(\alpha)}=\bigcup_{\beta<\alpha} R^{(\beta)}$ if $\alpha$ is a limit ordinal,

(3) $R^{(\alpha)}=\left(K_{\alpha-1}\right)^{\prime} \cap R$ if $\alpha$ is not a limit ordinal, $\alpha>0$.

It follows that $R^{(\beta)} \subset R^{(\alpha)}$ and $K_{\beta} \subset K_{\alpha}$ if $\beta \leqq \alpha$. Also $K_{0}=R$ and $K_{\delta}=R\left(R^{*}\right)^{-1}$ is the right quotient field of $R$. If $I$ is an interval of ordinals, $I^{*}$ will denote the set of nonlimit ordinals of $I$. For each $\alpha \in(0, \delta]^{*}$ an element $z \in R$ is called an $\alpha$-prime if $z R$ is maximal in $\left\{z R \mid z \in R \backslash R^{(\alpha-1)}\right\}$. Evidently, $\alpha$-primes exist because of the acc for right ideals of $R$; 1-primes are the usual primes and if $\alpha>1, \alpha$-primes have infinite dimension in $R$ and are primes in $K_{\alpha-1}$. The details regarding the construction of the $D$-chain may be found in [1].

We now define the (right) local skew degree of $R$ to be the least ordinal $\lambda$ such that $K_{\lambda}$ is a local ring. This definition is valid since $K_{\delta}$ is itself a local ring.

Our main result is the following Theorem whose proof will follow from the two lemmas below.

THEOREM. Let $R$ be a PRI domain with right $D$-chain $\left\{R^{(\alpha)} \mid 0 \leqq \alpha \leqq \delta\right\}$, let $K_{\alpha}=R\left(R^{(\alpha)}\right)^{-1}$, and let $\lambda$ be the local skew degree of $R$. Then $K_{\alpha}$ is a local ring for each $\alpha \geqq \lambda$. Equivalently, $R$ has a unique $(\alpha+1)$-prime for $\delta>\alpha \geqq \lambda$.

In what follows the term "unique" means unique up to right unit factor.

Lemma 1. Suppose $R$ has a unique $\beta$-prime for some $\beta \in[1, \delta]^{*}$. Then $R$ has a unique $\alpha$-prime for each $\alpha \in[\beta, \delta]^{*}$.

Proof. Proceeding by transfinite induction let $\alpha \in(\beta, \delta]^{*}$ and assume $R$ has a unique $\sigma$-prime whenever $\sigma \in[\beta, \alpha)^{*}$. To show $R$ has a unique $\alpha$-prime let $y$ and $z$ be two $\alpha$-primes in $R$ with $y R \neq z R$; we seek a contradiction. Let $d R=y R+z R$. We claim that if $\sigma \in[\beta, \alpha)^{*}$ then $d \in R \backslash R^{(\sigma)}$ 
For suppose $\sigma \in[\beta, \alpha)^{*}$ and let $x$ be the unique $\sigma$-prime in $R$. Now $y R \subset$ $x R$ by the maximum condition on the right ideals of $R$. In fact, $y R \subset$ $\bigcap_{n=0}^{\infty} x^{n} R$; otherwise we would have $y=x^{k} s$ where $k$ is the largest such integer and $s \in R \backslash x R$; it would then follow that $s \in R^{(\sigma-1)}$, otherwise $s R \subset$ $x R$ by the maximum condition and therefore $y \in R^{(\sigma)}$ which contradicts the fact that $y$ is an $\alpha$-prime. Similarly we have $z R \subset \bigcap_{n=0}^{\infty} x^{n} R$ and therefore $d R \subset \bigcap_{n=0}^{\infty} x^{n} R$. Since $x$ is prime in $K_{\sigma-1}$, the dimension of $d$ in $K_{\sigma-1}$ is infinite; that is, $d \notin\left(K_{\sigma-1}\right)^{\prime}$ and so $d \in R \backslash R^{(\sigma)}$. This establishes the claim. Now $y R \subsetneq d R$ implies $d \in R^{(\alpha-1)}$ because $y$ is an $\alpha$-prime. If $\alpha-1$ is not a limit ordinal then we have contradicted the claim. If $\alpha-1$ is a limit ordinal then $\beta<\alpha-1$ because $\beta$ is not a limit ordinal. Hence $R^{(\alpha-1)}=\bigcup_{\sigma<\alpha-1} R^{(\sigma)}$ and we may restrict the union to $\sigma \in[\beta, \alpha-1)^{*}$. Thus we may choose $\sigma \in[\beta, \alpha-1)^{*}$ such that $d \in R^{(\sigma)}$ which again contradicts the claim. We conclude $y R=z R$ which contradicts our original supposition.

LeMma 2. For each $\alpha<\delta, K_{\alpha}$ is a local ring iff $R$ has a unique $(\alpha+1)$ prime.

Proof. If $K_{\alpha}$ is a local ring then $R \backslash R^{(\alpha)}$ is an ideal, say $R \backslash R^{(\alpha)}=x R$. Then $x$ is clearly the unique $(\alpha+1)$-prime of $R$. Conversely, assume that $R$ has a unique $(\alpha+1)$-prime $x$, let $z$ be any prime in $K_{\alpha}$ and choose $z_{1} \in R$ such that $z_{1} R=z K_{\alpha} \cap R$. Then $z_{1} K_{\alpha}=z K_{\alpha}$ and $z_{1} R \subset x R$ by the maximum condition for right ideals of $R$. Therefore $z K_{\alpha} \subset x K_{\alpha}$ which means $z K_{\alpha}=x K_{\alpha}$ since $z$ and $x$ are primes in $K_{\alpha}$. This shows that $K_{\alpha}$ is a PRI domain with a unique prime. Consequently $K_{\alpha}$ is a local ring.

Proof of THE Theorem. If the local skew degree $\lambda$ of $R$ is equal to $\delta$ then there is nothing to prove. Assume $\lambda<\delta$. Since $K_{\lambda}$ is a local ring, $R$ has a unique $(\lambda+1)$-prime. If $\alpha>\lambda$, then $R$ has a unique $(\alpha+1)$-prime by Lemma 1 so that $K_{\alpha}$ is a local ring by Lemma 2 .

In conclusion we refer the reader to [1] for examples of PRI domains with right $D$-chain of any preassigned length $\delta$.

\section{REFERENCES}

1. R. A. Beauregard, Infinite primes and unique factorization in a principal right ideal domain, Trans. Amer. Math. Soc. 141 (1969), 245-253. MR 39 \#4206.

Department of Mathematics, University of Rhode Island, Kingston, Rhode ISLAND 02881 